\title{
A TRANSLATION THEOREM FOR ANALYTIC FEYNMAN INTEGRALS $\left({ }^{1}\right)$
}

\author{
BY \\ R. H. CAMERON AND D. A. STORVICK
}

1. Introduction. It is the purpose of this paper to obtain a translation theorem for analytic Feynman integrals based on the following translation theorem for Wiener integrals given in [2] and [3] $\left({ }^{2}\right)$.

THEOREM T. Let $x_{0}(t)$ be a real absolutely continuous function on $[a, b]$ which vanishes at $t=a$, and let $x_{0}^{\prime}(t)$ be essentially $\left({ }^{3}\right)$ of bounded variation $\left({ }^{4}\right)$ on $[a, b]$. Let $C[a, b]$ be the space of real continuous functions $x(t)$ on $[a, b]$ that vanish at $t=a$, and let $G(x)$ be a Wiener measurable functional on $C[a, b]$. Then if either member of the following equation exists, both exist and they are equal:

(1)

$$
\int_{C[a, b]} G\left(x+x_{0}\right) d x
$$

$$
=\exp \left(-\frac{1}{2} \int_{a}^{b}\left[x_{0}^{\prime}(t)\right]^{2} d t\right) \int_{C[a, b]} G(x) \exp \left(\int_{a}^{b} x_{0}^{\prime}(t) d x(t)\right) d x
$$

The analytic Wiener integral and analytic Feynman integral were defined in [1] by analytic extension from the Wiener integral as follows.

Definition. Let the complex number $\lambda_{0}$ satisfy $\operatorname{Re} \lambda_{0} \geqq 0$ and $\lambda_{0} \neq 0$, so that $\lambda_{0}=\left|\lambda_{0}\right| \exp (i \theta)$ for some $\theta$ on the interval $[-\pi / 2, \pi / 2]$. Let $F(x)$ be a functional defined on $C[a, b]$ and such that the Wiener integral

$$
J(\lambda) \equiv \int_{C[a, b]} F\left(\lambda^{-1 / 2} x\right) d x
$$

Presented to the Society, September 1, 1965; received by the editors August 25, 1965.

(1) Research sponsored by the Air Force Office of Scientific Research, Office of Aerospace Research, United States Air Force, Grant No. AF-AFOSR-381-63.

$\left(^{2}\right)$ Formula (1) given here differs slightly from those in [2] and [3] in that $[a, b]$ is used instead of $[0,1]$ and the Wiener integrals are normalized on the basis of "unit variance per unit time" instead of "half unit variance per unit time."

$\left(^{3}\right)$ The integral $\int_{a}^{b} x_{0}^{\prime}(t) d x(t)$ in (1) denotes the Riemann-Stieltjes integral obtained by replacing $x_{0}^{\prime}(t)$ by a function of bounded variation which is equal to $x_{0}^{\prime}(t)$ almost everywhere.

$\left.{ }^{4}\right)$ This restriction on $x_{0}^{\prime}(t)$ may be weakened to $x_{0}^{\prime}(\cdot) \in L_{2}$ provided that we interpret $\int_{a}^{b} x_{0}^{\prime}(t) d x(t)$ in the sense of [5]. 
exists for all real $\lambda$ in the interval $\left|\lambda_{0}\right|<\lambda<\left|\lambda_{0}\right|+\delta$ for some $\delta>0$. Then if $J(\lambda)$ can be extended so that it is defined and continuous on the closed region $\left({ }^{5}\right)$

$$
S=\left\{\lambda: \lambda=\rho e^{i \gamma},\left|\lambda_{0}\right| \leqq \rho \leqq\left|\lambda_{0}\right|+\left(1-\gamma \theta^{-1}\right) \delta, \gamma \in[0, \theta], \text { or } \gamma \in[\theta, 0]\right\}
$$

and analytic in its interior, we define

$$
\int_{C[a, b]}^{\mathrm{anw_{ \lambda _{0 } }}} F(x) d x=J\left(\lambda_{0}\right)
$$

and we call the left member of (4) the "analytic Wiener integral of $F(x)$ with revar parameter $\lambda_{0}$."

In particular, if $\lambda_{0}=-i q$ where $q$ is real, the integral (4) will be called the "analytic Feynman integral with parameter $q$," and we write

$$
\int_{C[a, b]}^{\mathrm{anf}_{q}} F(x) d x=\int_{C[a, b]}^{\mathrm{anw}-\mathrm{ta}} F(x) d x .
$$

If $q=1$, we shall write simply anf instead of $\mathrm{anf}_{1}$.

For use in the present paper we define uniform analytic Wiener (and Feynman) integrability.

Definition. Let $\lambda_{0}$ and $\theta$ be given as in the previous definition, let $A$ be any nonempty abstract set, and let $F(x \mid \alpha)$ be a functional defined on $C[a, b] \times A$. Suppose that there is a positive $\delta$ (independent of $\alpha$ ) and a corresponding set $S$ given by (3) and a function $J(\lambda \mid \alpha)$ defined on $S \times A$ having the following properties: $J(\lambda \mid \alpha)$ is analytic in $\lambda$ in the interior of $S$ for each fixed $\alpha \in A$ and $J(\lambda \mid \alpha)$ is uniformly continuous in $\lambda$ on $S$ uniformly with respect to $\alpha$ for all $\alpha$ in $A$, and finally,

$$
J(\lambda \mid \alpha)=\int_{C[a, b]} F\left(\lambda^{-1 / 2} x \mid \alpha\right) d x
$$

for all real $\lambda$ in the interval $\left|\lambda_{0}\right|<\lambda<\left|\lambda_{0}\right|+\delta$ and for all $\alpha$ in $A$. Then we say that $F(x \mid \alpha)$ is "analytic Wiener integrable with revar parameter $\lambda_{0}$ uniformly with respect to $\alpha$ over $A$." In particular, if $\lambda_{0}=-i q$ where $q$ is real, we say that $F(x \mid \alpha)$ is "analytic Feynman integrable with parameter $q$ uniformly with respect to $\alpha$ over $A$." In particular, if the parameter $q$ is not mentioned, it is understood to be equal to unity.

It is of course clear that uniform analytic Wiener (or Feynman) integrability implies analytic Wiener (or Feynman) integrability.

Now in order to obtain a translation theorem for analytic Feynman integrals, we will need to use a theorem on analytic extension of a function of several complex variables which the authors proved in [4]. (That paper was in fact motivated by

(5) If $\theta=0$, interpret $S$ to be the real interval $\left[\lambda_{0}, \lambda_{0}+\delta\right]$, omit the analyticity requirement since the interior of $S$ is empty, and define the analytic Wiener integral to be $J\left(\lambda_{0}+\right)$. 
the need to use it in this one.) We now state a special case of the main theorem of that paper for use below.

THEOREM A. Let $\Lambda$ be a simply connected region of the complex $\lambda$-plane whose intersection with the real axis is a nonempty interval $l$. Let $M$ denote the entire complex $\mu$-plane and let $m$ be the real axis in this plane. Let $\Phi(\lambda, \mu)$ be defined on $\Omega \equiv(\Lambda \times m) \cup(l \times M)$, and let it be bounded in every compact subset of $\Omega$. Further let $\Phi$ be an analytic function of $\lambda$ over $\Lambda$ for each $\mu$ in $m$, and let it be an entire function of $\mu$ for each $\lambda$ in l. Then it follows that there exists a function $\Phi^{*}(\lambda, \mu)$ which is defined and analytic in $(\lambda, \mu)$ everywhere on $\Lambda \times M$ and which satisfies

$$
\Phi^{*}(\lambda, \mu)=\Phi(\lambda, \mu)
$$

everywhere in $\Omega$.

By using Theorems $\mathrm{T}$ and $\mathrm{A}$ and the definitions given above, we shall obtain the following translation theorem for analytic Feynman integrals.

THEOREM 1. Let $\delta$ be a positive number $<1$, let $\Lambda$ be the region

$$
\Lambda=\left\{\lambda\left|\lambda=\rho e^{i \gamma},\right| \rho-1 \mid<\delta\left(1-\frac{2}{\pi}|\gamma|\right),-\frac{\pi}{2}<\gamma<\delta\right\}
$$

let $N$ be a neighborhood of $-i$ in the complex $\mu$-plane, and let $D$ be the unit disk $|\mu|<1$. Let $x_{0}(t)$ be as specified in Theorem $\mathrm{T}$, and let $F$ be a functional that satisfies the following two conditions:

$$
\int_{C[a, b]} F\left(\lambda^{-1 / 2} x\right) \exp \left(\mu \int_{a}^{b} x_{0}^{\prime}(t) d x(t)\right) d x
$$

exists for all real $\lambda$ in $1-\delta<\delta<1+\lambda$ and all complex $\mu$ and is bounded on all compact subsets of this three dimensional region; and

$$
\int_{C[a, b]}^{a n w_{\lambda}} F(x) \exp \left(\mu \int_{a}^{b} x_{0}^{\prime}(t) d x(t)\right) d x
$$

exists for all $\lambda$ in $\Lambda$ and all real $\mu$ and is bounded on all compact subsets of this three dimensional region.

Then it follows that the analytic Wiener integral in (ii) exists and is analytic in $(\lambda, \mu)$ for all $\lambda$ in $\Lambda$ and all complex $\mu$. Moreover if $F$ also satisfies the third condition

$$
\int_{C[a, b]}^{\mathrm{anf}} F(x) \exp \left(\mu \int_{a}^{b} x_{0}^{\prime}(t) d x(t)\right) d x
$$


exists uniformly with respect to $\mu$ over $N \cap D$ and approaches a limit as $\mu \rightarrow-i$ from inside $D$, then the following integrals exist and equality holds:

(7)

$$
\begin{aligned}
& \int_{C[a, b]}^{\mathrm{anf}} F\left(x+x_{0}\right) d x \\
& \quad=\exp \left(\frac{i}{2} \int_{a}^{b}\left[x_{0}^{\prime}(t)\right]^{2} d t\right) \int_{C[a, b]}^{\mathrm{anf}} F(x) \exp \left(-i \int_{a}^{b} x_{0}^{\prime}(t) d x(t)\right) d x .
\end{aligned}
$$

Since the existence of the integral in (i) follows from the existence of the integral in (ii), condition (i) may obviously be replaced by the stronger condition (iv) below.

COROLlaRY. Theorem 1 still holds if condition (i) is replaced by condition (iv):

$$
\left[\sup _{1-\delta<\lambda<1+\delta}\left|F\left(\lambda^{-1 / 2} x\right)\right|\right] \exp \left(\mu \int_{a}^{b} x^{\prime}(t) d x(t)\right)
$$

is Wiener integrable over $C[a, b]$ for each real $\mu$.

2. The proof of Theorem 1. Let us first assume that conditions (i) and (ii) are satisfied, and let the integrals given in (i) and (ii) be denoted by $\Psi(\lambda, \mu)$ and $\Phi(\lambda, \mu)$ respectively. Then by the definition of the analytic Wiener integral, we have when $\lambda$ and $\mu$ are both real and $|\lambda-1|<\delta$,

$$
\Phi(\lambda, \mu)=\Psi\left(\lambda, \lambda^{-1 / 2} \mu\right) .
$$

Now let

$$
\tilde{\Phi}(\lambda, \mu)= \begin{cases}\Phi(\lambda, \mu), & \text { for } \lambda \in \Lambda \text { and } \mu \text { real, } \\ \Psi\left(\lambda, \lambda^{-1 / 2} \mu\right), & \text { for } \lambda \text { real and }|\lambda-1|<\delta \text { and } \mu \text { complex. }\end{cases}
$$

By the definition of $\Phi$ in (ii) and the monodromy theorem, we see that $\Phi(\lambda, \mu)$ is analytic in $\lambda$ throughout $\Lambda$ when $\lambda$ is not real, and since $\Phi$ is continuous in $\lambda$ on the real axis, we have analyticity throughout the whole of $\Lambda$ for each real $\mu$. The same statements of course apply to $\tilde{\Phi}$ since it equals $\Phi$ when $\mu$ is real. Moreover $\Psi(\lambda, \mu)$ given by (i) is clearly an entire function of $\mu$ for each real $\lambda$ in $|\lambda-1|<\delta$, and this is also true for $\tilde{\Phi}(\lambda, \mu)$. Thus it is clear that $\tilde{\Phi}$ satisfies the hypotheses of Theorem $\mathrm{A}$, and hence there exists a function $\Phi^{*}(\lambda, \mu)$ which is defined and analytic in $(\lambda, \mu)$ on $\Lambda \times M$ and which equals $\tilde{\Phi}(\lambda, \mu)$ wherever the latter is defined; (here, as in Theorem A, $M$ denotes the complex $\mu$-plane). In particular,

$$
\Phi^{*}(\lambda, \mu)=\Phi(\lambda, \mu), \quad \text { for } \lambda \in \Lambda \text { and } \mu \text { real }
$$

and

$$
\Phi^{*}(\lambda, \mu)=\Psi\left(\lambda, \lambda^{-1 / 2} \mu\right), \quad \text { for } \lambda \text { real and }|\lambda-1|<\delta \text { and } \mu \in M \text {. }
$$


Now since for each complex $\mu,(10)$ holds for real $\lambda$ on $|\lambda-1|<\delta$ and the Wiener integral represented by $\Psi\left(\lambda, \lambda^{-1 / 2} \mu\right)$ exists, and since $\Phi^{*}(\lambda, \mu)$ represents an analytic extension of this into the appropriate horn-shaped region, the analytic Wiener integral indicated by (ii) exists in $\Lambda \times M$ and is equal to $\Phi^{*}(\lambda, \mu)$. Thus (9) holds over all of $\Lambda \times M$ if we extend the definition of $\Phi$ as the integral given in (ii) over all of $\Lambda \times M$. Moreover $\Phi$ is analytic over $\Lambda \times M$, and the first conclusion of the theorem is proved.

Now assume that condition (iii) is also satisfied. Then by (iii) there is a set $S$ given by (3) with $\lambda_{0}=-i$ and some $\delta$ (not necessarily the same as the one mentioned in the hypothesis of Theorem 1) such that $\Psi\left(\lambda, \lambda^{-1 / 2} \mu\right)$ has for each $\mu$ in $N \cap D$ an extension which is continuous in $\lambda$ throughout $S$ and analytic in the interior of $S$. Moreover this extension is continuous in $\lambda$ over $S$ uniformly for $\mu \in N \cap D$. Now this extension must of course be equal to $\Phi(\lambda, \mu)$ at their common points of definition. But if the $\delta$ associated with $S$ is taken sufficiently small, $S-\Lambda$ consists of the one point $\lambda=-i$. Thus if we denote the integral in (iii) by $\Phi(-i, \mu)$, we have

$$
\Phi(-i, \mu)=\lim _{\lambda \rightarrow-i, \lambda \in S} \Phi(\lambda, \mu)
$$

uniformly in $\mu$ for $\mu \in N \cap D$. Moreover, by hypothesis (iii), we know that

$$
\lim _{\mu \rightarrow-i, \mu \in D} \Phi(-i, \mu)=L
$$

exists. (Here we are using the left member to define the right member.) Thus by the Moore-Smith theorem on repeated limits,

$$
\lim _{\lambda \rightarrow-i \text { over } S: \mu \rightarrow-i \text { over } D} \Phi(\lambda, \mu)=L .
$$

Also for $\lambda \in \Lambda$, (and hence for $\lambda \in S, \lambda \neq-i$ ), $\Phi(\lambda, \mu)$ is continuous in $\mu$ for all $\mu$. Thus for each $\lambda \in S, \lambda \neq-i$,

$$
\lim _{\mu \rightarrow-i \text { over } D} \Phi(\lambda, \mu)=\Phi(\lambda,-i),
$$

and by the Moore-Smith theorem

$$
\lim _{\lambda \rightarrow-i \text { over } S} \Phi(\lambda,-i)=L .
$$

Thus since $\Phi(\lambda,-i)$ is continuous in $\lambda$ over $\Lambda$, it is continuous in $\lambda$ over $S$ if it is given the value $L$ at $\lambda=-i$. But this is an extension of $\Psi\left(\lambda,-i \lambda^{-1 / 2}\right)$ which is analytic throughout the interior of $S$ and continuous over $S$. It follows from the definition of an analytic Feynman integral that (ii) is defined at $\mu=-i$ and

$$
\int_{C[a, b]}^{a n f} F(x) \exp \left(-i \int_{a}^{b} x_{0}^{\prime}(t) d x(t)\right) d x=L,
$$

and in accordance with (ii) we may properly write

$$
L=\Phi(-i,-i)
$$


Let us now apply Theorem $\mathrm{T}$ to the functional

$$
G(x)=F\left(\lambda^{-1 / 2} x\right)
$$

and at the same time replace $x_{0}$ by $\lambda^{1 / 2} x_{0}$. We obtain

$$
\begin{aligned}
& \int_{C[a, b]} F\left(\lambda^{-1 / 2} x+x_{0}\right) d x \\
& \quad=\exp \left(-\frac{\lambda}{2} \int_{a}^{b}\left[x_{0}^{\prime}(t)\right]^{2} d t\right) \int_{C[a, b]} F\left(\lambda^{-1 / 2} x\right) \exp \left(\lambda^{1 / 2} \int_{a}^{b} x_{0}^{\prime}(t) d x(t)\right) d x
\end{aligned}
$$

for real $\lambda$ on $|\lambda-1|<\delta$, since the right member exists and this implies the measurability of $F\left(\lambda^{-1 / 2} x\right)$. But the right member is the product of an entire function with $\Psi\left(\lambda, \lambda^{1 / 2}\right)$ which equals $\Phi(\lambda, \lambda)$. Thus for real $\lambda$ on $|\lambda-1|<\delta$, we have

$$
\int_{C[a, b]} F\left(\lambda^{-1 / 2} x+x_{0}\right) d x=\exp \left\{-\frac{\lambda}{2} \int_{a}^{b}\left[x_{0}^{\prime}(t)\right]^{2} d t\right\} \Phi(\lambda, \lambda) .
$$

Now since $\Phi(\lambda, \mu)$ is analytic in $\Lambda \times M, \Phi(\lambda, \lambda)$ is analytic in $\Lambda$. Thus $\Phi(\lambda, \lambda)$ is analytic in the interior of $S$ and continuous throughout $S$ except perhaps at $\lambda=-i$. But since $S-D$ contains only the point $-i$, (13) implies that

$$
\lim _{\lambda \rightarrow-i \text { over } S} \Phi(\lambda, \lambda)=L=\Phi(-i,-i)
$$

Thus the left member of (16) has an extension to $S$ which is continuous over $S$ and analytic in the interior of $S$. Hence the left member of (7) exists and we have

$$
\int_{C[a, b]}^{\mathrm{anf}} F\left(x+x_{0}\right) d x=\exp \left\{\frac{i}{2} \int_{a}^{b}\left[x_{0}^{\prime}(t)\right]^{2} d t\right\} \Phi(-i,-i) .
$$

Then (7) follows from (14), (15), (18); and Theorem 1 is proved.

\section{BIBLIOGRAPHY}

1. R. H. Cameron, The Ilstow and Feynman integrals, J. Analyse Math. 10 (1962-1963), 287-361.

2. R. H. Cameron and R. E. Gravies, Additive functionals on a space of continuous functions. I, Trans. Amer. Math. Soc. 70 (1951), 160-176.

3. R. H. Cameron and W. T. Martin, Transformations of Wiener integrals under translations, Ann. of Math. 45 (1944), 386-396.

4. R. H. Cameron and D. A. Storvick, Analytic continuation for functions of several variables, Trans. Amer. Math. Soc. 125 (1966), 7-12.

5. R. E. A. C. Paley, N. Wiener and A. Zygmund, Notes on random functions, Math. Z. 37 (1933), 647-668.

\section{UNIVERSITY OF MiNNESOTA,} MinNEAPOLIS, MinNesota 